sichtigen. ${ }^{105}$ Zur Vermeidung von Rechtsunsicherheit für die Schifffahrt und zur Reduzierung der Gefahr einer Umgehung von Umweltstandards sollten bundesweit einheitliche Standards angestrebt werden. In jedem Fall ist ein Einbringen der Biomasse in das Wasser verboten, weshalb diese im Einklang mit dem KrWG an Land verwertet oder entsorgt werden muss.

Open Access. Dieser Artikel wird unter der Creative Commons Namensnennung 4.0 International Lizenz veröffentlicht, welche die Nutzung, Vervielfältigung, Bearbeitung, Verbreitung und Wiedergabe in jeglichem Medium und Format erlaubt, sofern Sie den/die ursprünglichen Autor(en) und die Quelle ordnungsgemäß nennen, einen Link zur Creative Commons Lizenz beifügen und angeben, ob Änderungen vorgenommen wurden.

Die in diesem Artikel enthaltenen Bilder und sonstiges Drittmaterial unterliegen ebenfalls der genannten Creative Commons Lizenz, so- fern sich aus der Abbildungslegende nichts anderes ergibt. Sofern das betreffende Material nicht unter der genannten Creative Commons Lizenz steht und die betreffende Handlung nicht nach gesetzlichen Vorschriften erlaubt ist, ist für die oben aufgeführten Weiterverwendungen des Materials die Einwilligung des jeweiligen Rechteinhabers einzuholen.

Weitere Details zur Lizenz entnehmen Sie bitte der Lizenzinformation auf http://creativecommons.org/licenses/by/4.0/deed.de.

Open Access funding enabled and organized by Projekt DEAL.

105) Vgl. zum insoweit einschlägigen Naturschutzrecht Schatz, Die Regulierung der Einführung invasiver gebietsfremder Arten durch Biofouling an der Schnittstelle zwischen Naturschutzrecht und Seeschifffahrtsrecht, NuR 2021, $361 \mathrm{ff}$.

\title{
https://doi.org/10.1007/s10357-021-3914-3 \\ Der Abschneidewert für Stickstoffeinträge im Habitatschutz
}

\author{
Friedrich Hacker, Florian Jansen, Thomas Krämerkämper, Peter Kremer, Dirk Tessmer
}

๑๐ Der/die Autor(en) 2021. Dieser Artikel ist eine Open-Access-Publikation.

Die Auswirkungen von Stickstoffeinträgen in FFH-Gebiete sind eine der zentralen fachlichen und rechtlichen Fragestellungen im Habitatschutz. Das BVerwG hat hierzu - auf der Grundlage fachlicher Leitfäden - seine Rechtsprechung zu Bagatell-/Irrelevanzschwellen und einem Abschneidekriterium entwickelt. Während Bagatell-/Irrelevanzschwellen an der Empfindlichkeit von Lebensräumen orientiert sind, ist das Abschneidekriterium (in Höhe von $0,3 \mathrm{~kg} \mathrm{~N} /($ ha $\star a)$ ) ein $\mathrm{ab}-$ soluter Wert; liegt der Eintrag aus einem Projekt darunter, muss nicht weiter geprüft werden. Auch summierende Einträge unterhalb dieser Schwelle werden nicht berücksichtigt.

Im Folgenden wird - nach einem Überblick über die Rechtsprechung - gezeigt, dass die naturwissenschaftlichen Annahmen, die der rechtlichen Einordnung des BVerwG zugrunde liegen, nicht haltbar sind. Teilweise sind die fachlichen Aussagen nicht hergeleitet oder nachgewiesen (z. B. die untere Wirkungsschwelle), teilweise sind sie falsch (z.B. die Untergrenze der Messbarkeit oder die fehlende Zuordenbarkeit zu einem Emittenten). Es wird weiter gezeigt, dass von Einträgen unterhalb von $0,3 \mathrm{~kg} \mathrm{~N} /\left(\mathrm{ha}^{\star} \mathrm{a}\right)$ schädliche Wirkungen ausgehen können, was dann im Einzelfall untersucht werden muss.

Ein absoluter Abschneidekriterium in Höhe von 0,3 kg N/ (ha ${ }^{\star}$ a) kann daher vor dem Hintergrund, dass im Habitatschutzrecht vernünftige Zweifel ausgeschlossen sein müssen, keinen Bestand haben. Die vom BVerwG herangezogenen Leitfäden können nicht (mehr) als Fachkonventionen angesehen werden.

\section{Dipl.-Biol. Friedrich Hacker, Diplom-Biologe,}

Greifswald, Deutschland

Prof. Dr. rer. nat Florian Jansen,

Prof. für Landschaftsökologie und Standortkunde, Uni Rostock, Rostock, Deutschland

Dr. rer. nat. Thomas Krämerkämper, Diplom-Physiker, Castrop-Rauxel, Deutschland

Peter Kremer, Rechtsanwalt und Fachanwalt für Verwaltungsrecht, Berlin, Deutschland

Dirk Tessmer, Rechtsanwalt,

Frankfurt a.M., Deutschland

\section{Stickstoffeinträge als naturschutzfachliche und rechtliche Herausforderung}

Mit seinem Globalen Bericht zum Zustand der Natur hat der Weltbiodiversitätsrat (IPBES) eindringlich darauf aufmerksam gemacht, dass weltweit bis zu einer Million Tier- und Pflanzenarten vom Aussterben bedroht und zahlreiche Ökosysteme gefährdet sind. ${ }^{1}$ Die sich darin offenbarende Biodiversitätskrise ${ }^{2}$ macht selbst vor den an sich streng geschützten Gebieten des Netzes Natura 2000 nicht halt. Exemplarischen Beleg bieten dafür die Ergebnisse des FFH-Berichts 2019, die erkennen lassen, dass nur etwa $30 \%$ der dort geschützten natürlichen Lebensraumtypen einen günstigen Erhaltungszustand aufweisen, während sich $32 \%$ in einem ungünstig-unzureichenden und sogar $37 \%$ in einem ungünstig-schlechten Zustand befinden. ${ }^{3}$

Auch wenn verschiedene Gefährdungsfaktoren hierfür verantwortlich sind, ist die Belastung nährstoffempfindlicher Lebensraumtypen mit Stickstoff eine der wesentlichen Ursachen. ${ }^{4}$ Der Eintrag reaktiver Stickstoffverbindungen, der durch die Landwirtschaft, durch Verbrennungspro-

1) Helmholz-Zentrum für Umweltforschung GmbH (UFZ), Das „Globale Assessment" des Weltbiodiversitätsrates IPBES. Auszüge aus dem "Summary für policymakers“, Stand: 6.5.2019.

2) Bundesamt für Naturschutz (BfN), Agrar-Report 2017. Biologische Vielfalt in der Agrarlandschaft, Juni 2017, S. 6.

3) Sutton et al., 2011, The European nitrogen assessment: sources, effects, and policy perspectives, S. $463 \mathrm{f} . ;$ Bundesministerium für Umwelt, Naturschutz und nukleare Sicherheit (BMU), Die Lage der Natur in Deutschland. Ergebnisse von EU-Vogelschutz- und FFH-Bericht, 19.5.2020, S. 5 .

4) Vgl. nur Sachverständigenrat für Umweltfragen (SRU), Stickstoff: Lösungsstrategien für ein drängendes Umweltproblem, Sondergutachten 2015, S. 21; ders., Für einen flächenwirksamen Insektenschutz, Stellungnahme Oktober 2018, S. 22 f.; Finck/ Heinze/Raths/Riecken/Ssymank, Rote Liste der gefährdeten Biotoptypen Deutschlands, dritte fortgeschriebene Fassung 2017, Naturschutz und Biologische Vielfalt, Heft 156, 2017, S. 123 . 
zesse in der Industrie und bei der Stromerzeugung sowie durch den Straßenverkehr hervorgerufen wird, hat erheblichen Anteil an der besorgniserregenden Erhaltungssituation der in Anhang II FFH-RL aufgeführten Lebensraumtypen. Gerade die Stickstoffimmissionen aus der Luft sind für den dramatischen Rückgang der charakteristischen Arten der FFH-Lebensraumtypen verantwortlich; der Rückgang betrifft inzwischen auch die mittelhäufigen Arten. ${ }^{5}$ Anthropogene Stickstoffeinträge können bereits in geringen Dosen zu Eutrophierung und Versauerung empfindlicher Lebensraumtypen führen. ${ }^{6}$ Die vom Umweltbundesamt (UBA) im Jahre 2011 aufgeworfene Frage „Stickstoff - Zuviel des Guten?"7 nimmt an Brisanz - gerade wegen der Auswirkung auf Natura 2000-Gebiete - zu.

Die Bundesrepublik Deutschland ist nach der FFHRichtlinie verpflichtet, geeignete Maßnahmen zu ergreifen, damit die aus unterschiedlichen Quellen stammenden Stickstoffeinträge nicht zu einer Verschlechterung der Gebiete von gemeinschaftlicher Bedeutung führen und damit auch der günstige Erhaltungszustand dieser Gebiete nicht verhindert wird (Art. 6 Abs. 2, 3 FFH-RL). Das legt mit Blick auf den unzureichenden bzw. schlechten Zustand des weit überwiegenden Teils der zu schützenden Lebensraumtypen die zunächst vom BVerwG geteilte Annahme nahe, dass für zusätzliche Stickstoffbelastungen kein Raum bleibt. ${ }^{8}$ In der Folgezeit hat dieser Grundsatz allerdings insoweit eine Einschränkung erfahren, als projektbedingte Stickstoffeinträge stets und unabhängig von der Belastungssituation des betroffenen Lebensraumtyps irrelevant sein sollen, wenn die zusätzliche Belastung nicht mehr als $0,3 \mathrm{~kg} \mathrm{~N} /\left(\mathrm{ha}^{\star}\right.$ a) beträgt. ${ }^{9}$ Vor dem Hintergrund der Erkenntnis, dass lang anhaltende Stickstoffbelastungen selbst in geringen Dosen zu Schädigungen führen können, ist die Anerkennung eines solchen „, $A b$ schneidewertes", der seinen Niederschlag bereits in Stickstoffleitfäden ${ }^{10}$ und auch in der neuen TA Luft gefunden hat, ${ }^{11}$ in hohem Maße rechtfertigungsbedürftig. Dies auch angesichts dessen, dass die Bewahrung der Vielfalt der Arten und Lebensräume einem Gebot von Verfassungsrang entspricht (Art. 20a GG). ${ }^{12}$ Es besteht daher hinreichend Anlass, die Belastbarkeit des höchstrichterlichen Ansatzes aus rechtlicher und fachlicher Perspektive kritisch zu analysieren.

\section{Bagatell- und Abschneidewerte}

Die Beurteilung der Empfindlichkeit von Biotopen oder Lebensräumen gegenüber eutrophierend ${ }^{13}$ wirkenden Stickstoffeinträgen orientiert sich an der Frage, ob der sogenannte Critical Load überschritten wird. ${ }^{14}$ Der Critical Load wird als kritische Belastungsrate angesehen, bei dessen Einhaltung oder Unterschreitung es weder akut noch langfristig zu schädigenden Wirkungen auf empfindliche Okosysteme kommt. ${ }^{15}$ Empirisch ermittelte Critical Loads für unterschiedliche Lebensraumtypen enthält die sog. „Berner Liste“. ${ }^{16}$ Das BVerwG hat das Konzept der empirischen Critical Loads anerkannt. ${ }^{17}$

Die Rechtsprechung hat sich bereits früh mit der Frage beschäftigt, ob es eine Untergrenze für Stickstoffeinträge gibt, bei deren Unterschreitung eine Beeinträchtigung ausgeschlossen und deshalb nicht geprüft werden muss. ${ }^{18}$

Zunächst anerkannt wurde ein Bagatell- oder Irrelevanzwert, der - relativ zur Empfindlichkeit des Schutzguts bei 3\% des Critical Loads von der Rechtsprechung identifiziert wurde und für den Stickstoffeinträge aus anderen Projekten kumulativ zu berücksichtigen sind. ${ }^{19}$ Dieser Ansatz ist nicht Gegenstand dieses Beitrags.

Neben diesem Bagatell- oder Irrelevanzwert setzt die Rechtsprechung einen absoluten Abschneidewert in Höhe von $0,3 \mathrm{~kg} \mathrm{~N} /(\mathrm{ha} \star \mathrm{a})$ an. ${ }^{20}$ Stickstoffeinträge bis $0,3 \mathrm{~kg}$ pro Hektar und Jahr sind demnach nicht zu berücksichtigen. Sie kennzeichnen, so das BVerwG, die Grenzen der Flä- che, auf der projektbedingte Stickstoffeinträge eine Rolle spielen. Jenseits einer 0,3 kg-Isolinie gelten Stickstoffeinträge als unbeachtlich, unabhängig von der Art und dem Zustand betroffener Lebensräume, der Höhe der Vorbelastung oder kumulativer Einwirkungen aus anderen Projekten. ${ }^{21}$

5) Jansen/Bonn/Bowler/Bruelheide/Eichenberg, 2019, Moderately common plants show highest relative losses. Conservation Letters. https://doi.org/10/gf8pp4.

6) Bund/Länderarbeitsgemeinschaft für Immissionsschutz (LAI)/BundLänderarbeitsgemeinschaft Naturschutz, Landschaftspflege und Erholung (LANA), Hinweise zur Prüfung von Stickstoffeinträgen in der FFH-Verträglichkeitsprüfung für Vorhaben nach dem Bundes-Immissionsschutzgesetz - Stickstoffleitfaden BImSchG-Anlagen -, 19. 2.2019, S. 1; Stickstoffleitfaden Straße 2019 (siehe unten Fn. 24), S. 13.

7) Umweltbundesamt (UBA), Stickstoff - Zuviel des Guten? Überlastung des Stickstoffkreislaufs zum Nutzen von Mensch und Umwelt reduzieren, Januar 2011.

8) BVerwG, Urt. v. 17.1.2007 - 9 A 20.05, NuR 2007, 336, Rdnr. 108; BVerwG, Beschl. v. 10.11.2009 - 9 B 28.09, NuR 2010, 190, Rdnr. 3.

9) Vgl. nur BVerwG, Urt. v. 15. 5.2019 - 7 C 27.17, NuR 2019, 763 , Rdnr. 31 ff.; BVerwG, Urt. v. 21.1.2021 - 7 C 9.19, Rdnr. 29, juris.

10) Nachweise in Fn. 23 und 24.

11) Gemeinsames Ministerialblatt, Ausgabe 48-54/2021, S. $1050 \mathrm{ff}$., Anhang 8, S. 1186

12) Vgl. nur BVerfG, Urt. v. 24.11.2010 - 1 BvF 2/05, NuR 2011, 39, Rdnr. 137; Gärditz, in: Landmann/Rohmer, Umweltrecht I, Stand: 95. Lfg. 2021, Nr. 3 Art. 20a, Rdnr. 9; Jarass, Grundgesetz, 15. Aufl. 2018, Art. 20a, Rdnr. 3; Murswiek, in: Sachs, Grundgesetz, 9. Aufl. 2021, Art. 20a, Rdnr. 30; BVerfG, Beschl. v. 24.3.2021 - 1 BvR 2656/18 u. a., juris.

13) Von Stickstoffeinträgen geht auch eine versauernde Wirkung aus, die nicht Gegenstand dieser Abhandlung ist; die rechtlichen Fragen sind jedoch vergleichbar.

14) Der Critical Load bezieht sich auf die Deposition von Stickstoff in einer Pflanzengesellschaft. Zusätzlich muss auch die Konzentration stickstoffhaltiger Verbindungen in der Luft als mögliche Ursache einer Schädigung - über einen anderen Wirkweg - berücksichtigt werden. Die Grenze einer schädlichen Konzentration wird als „Critical Level“ bezeichnet. Siehe hierzu auch unten 6.2.1 und Fn. 63. Die Critical Levels sind nicht Gegenstand dieser Abhandlung.

15) Definition aus Umweltbundesamt, Glossar, https://www.umweltbundesamt.de/service/glossar/c?tag=Critical_Loads\#alphabar, abgerufen am 15.9.2021. Das BVerwG, Urt. v. 23.4.20149 A 25.12, NuR 2014, 706, Rdnr. 35 formuliert zurückhaltender (,CL sollen naturwissenschaftlich begründete Belastungsgrenzen (...) umschreiben..."). Zur Frage, ob die Einhaltung des Critical Loads langfristig gewährleistet, dass es zu keinen Schäden kommt, siehe unten 6.2.3.

16) BVerwG, Urt. v. 23.4.2014 - 9 A 25.12, NuR 2014, 706 , Rdnr. 35: „Als empirische CL werden die im sogenannten ICPManual veröffentlichten Ergebnisse der Arbeiten der Arbeitsgruppe Bobbink bezeichnet, die auf Erfahrungen und Felduntersuchungen beruhen."

17) BVerwG, Urt. v. 12.3.2008 - 9 A 3.06, NuR 2008, 633 , Rdnr. 109; BVerwG, Urt. v. 14.4.2010 - 9 A 5.08, NuR 2010, 558, Rdnr. 87; BVerwG, Urt. v. 29.9.2011 - 7 C 21.09, NuR 2012, 119, Rdnr. 41; BVerwG, Urt. v. 18.3.2013 - 9 A 22.11, NuR 2013, 565, Rdnr. 65.

18) BVerwG, Beschl. v. 10.11.2009 - 9 B 28/09, NuR 2010, 190, Rdnr. 8, mit Hinweis auf den Verhältnismäßigkeitsgrundsatz im damaligen Art. 5 Abs. 3 EG, jetzt Art. 5 Abs. 4 EUV.

19) BVerwG, Urt. v. 14.4.2010 - 9 A 5.08 - NuR 2010, 558 , Rdnr. 93; BVerwG, Beschl. v. 5. 9.2012 - 7 B 24.12, NuR 2012, 784, Rdnr. 9; BVerwG, Urt. v. 6.11.2012 - 9 A 17.11, NuR 2014, 344, Rdnr. 62 und 93.

20) Zur Abgrenzung von Bagatell- bzw. Irrelevanzschwelle einerseits und Abschneidekriterium andererseits siehe BVerwG, Urt. v. 21.1.2021 - 7 C 9.19, Rdnr. 29ff., juris.

21) BVerwG, Urt. v. 15.5.2019 - 7 C 27.17, NuR 2019, 763 , Rdnr. 33. 


\section{Die Rechtsprechung zum Abschneidekriterium}

Zur Herleitung des Abschneidewerts ${ }^{22}$ stützt sich das BVerwG im Wesentlichen auf den FE-Bericht Stickstoff ${ }^{23}$ aus dem Jahr 2013, dem darauf beruhenden Stickstoffleitfaden Straße ${ }^{24}$ aus dem Jahr 2019, und einen Aufsatz zu Stickstoffeinträgen in der FFH-Verträglichkeitsprüfung. ${ }^{25}$

Aus diesen Publikationen und unter Bezugnahme auf ältere Rechtsprechung leitet das BVerwG folgende Begründung für den Abschneidewert her:

Unterhalb einer Schwelle von $0,3 \mathrm{~kg} \mathrm{~N} /(\mathrm{ha} \star \mathrm{a})$ sei die $\mathrm{zu}-$ sätzliche von einem Vorhaben ausgehende Belastung nicht mehr mit vertretbarer Genauigkeit bestimmbar bzw. nicht mehr eindeutig von der vorhandenen Hintergrundbelastung abgrenzbar. ${ }^{26}$ Es ließen sich auch keine kausalen $\mathrm{Zu}-$ sammenhänge zwischen Emission und Deposition nachweisen. $\$ 34$ BNatSchG fordere aber einen Zusammenhang zwischen Stickstoffeintrag eines Vorhabens und Beeinträchtigung. ${ }^{27}$ Empirische Untersuchungen entlang viel belasteter Straßen hätten außerhalb der mithilfe des 3\%-Kriteriums ermittelten Flächen bisher keine signifikanten schädlichen Effekte von stickstoffhaltigen Immissionen der Straße auf die Vegetation ergeben. ${ }^{28}$

Der Auffassung des OVG Münster, dass wegen der Pflicht zur Berücksichtigung kumulativer Wirkungen der Abschneidewert auf $0,05 \mathrm{~kg} \mathrm{~N} /\left(\mathrm{ha}^{\star} \mathrm{a}\right)$ gesenkt werden müsse ${ }^{29}$, ist das BVerwG mit dem Argument fehlender Messbarkeit und fehlender Zuordenbarkeit nicht gefolgt. ${ }^{30}$

Zusätzlich argumentiert das BVerwG, dass der Wert von $0,3 \mathrm{~kg} \mathrm{~N} /(\mathrm{ha} \star \mathrm{a})$ deutlich unterhalb einer sicheren Ursache-Wirkungs-Relation liege, weil die meisten experimentellen wissenschaftlichen Studien zu den Einflüssen zusätzlicher Stickstoffeinträge auf die Vegetation mit Stickstoffabgaben in Stufen von mindestens 5 bis $10 \mathrm{~kg} \mathrm{~N} /\left(\mathrm{ha}^{\star} \mathrm{a}\right)$ arbeiteten.

Ein Abschneidewert in den Niederlanden beschäftigte zwischenzeitlich den EuGH. ${ }^{31}$ Im Rahmen eines vorsorgenden bzw. kontingentierenden Stickstoffreduzierungsprogramms wurde in den Niederlanden ein Abschneidewert in Höhe von $14 \mathrm{~g} \mathrm{~N} /\left(\mathrm{ha}^{\star} \mathrm{a}\right)$ festgesetzt, bei dessen Unterschreitung die Prüfung stickstoffbedingter Beeinträchtigungen entfallen könne. Der EuGH stellt fest, dass ein solcher Abschneidewert nur zulässig ist, soweit sich die zuständigen Behörden Gewissheit verschafft haben, dass aus wissenschaftlicher Sicht kein vernünftiger Zweifel daran bestehe, dass keines der erlaubten Projekte schädliche Auswirkungen auf das betreffende Gebiet habe. Das BVerwG sieht in der vom EuGH für $14 \mathrm{~g} \mathrm{~N} /\left(\right.$ ha $\left.{ }^{\star} \mathrm{a}\right)$ verlangten Prüfpflicht keinen Hinweis darauf, dass die deutsche Schwelle von $0,3 \mathrm{~kg} \mathrm{~N} /\left(\mathrm{ha}^{\star} \mathrm{a}\right)$ zu hoch sein könnte, da es in den Niederlanden um einen anderen Streitgegenstand gegangen sei, nämlich ein Programm, das einen Puffer für neue Stickstoffeinträge ermöglichen solle. ${ }^{32}$

Eine ausführliche Auseinandersetzung mit dem Abschneidekriterium enthält ein Urteil des BVerwG aus dem Jahr 2019. ${ }^{33}$ Der 9. Senat attestiert dem Stickstoffleitfaden Straße (Endversion 2019), der auf dem Forschungsbericht 2013 beruht, die Eigenschaft einer Fachkonvention, die den aktuell besten wissenschaftlichen Erkenntnisstand widerspiegle. ${ }^{34}$. Der Stickstoffleitfaden sei „von einem Gremium fachkundiger Wissenschaftler in einem mehrjährigen Abstimmungsprozess unter Einbeziehung maßgeblicher Expertenkreise und Beteiligung der Öffentlichkeit erstellt (worden), wobei auch die Naturschutzverbände ihre Stellungnahmen abgegeben und Bedenken vorgebracht haben. Einbezogen in den Prozess waren auch die staatlichen Fachgremien der Bund/Länder-Arbeitsgemeinschaften Immissionsschutz (LAI) und Naturschutz, Landschaftspflege und Erholung (LANA), mit denen die Anwendbarkeit des Leitfadens für immissionsschutzrechtliche Vorhaben koordiniert wurde. Die im Leitfaden zusammengefassten "Hinweise" beruhen damit auf einem breiten wissenschaftlichen Konsens. Dafür, dass es derzeit bessere wissenschaftliche Erkenntnisse geben könnte, die geeignet wären, Methodik, Grundannahmen oder Schlussfolgerungen des Stickstoffleitfadens substantiell in Frage zu stellen oder gar zu widerlegen, gibt es keine Anhaltspunkte, so dass die Grenzen der gerichtlich möglichen und gebotenen Aufklärung und Kontrolle insoweit erreicht sind (vgl. dazu BVerfG, Beschl. v. 23. 10. $2018-1$ BvR 2523/13, 1 BvR 595/14, NVwZ 2019, 52 Rdnr. 28f.).“

Zur Begründung des Abschneidekriteriums verweist der 9. Senat auf die bisherige Rechtsprechung des BVerwG, wie sie oben bereits dargestellt wurde.

Neben der Messbarkeit der Einträge und der Abgrenzbarkeit von der Hintergrundbelastung müsse sich der vorhabenbedingte Eintrag vor dem Hintergrund der mit der Ermittlung der Gesamtbelastung verbundenen Unsicherheiten statistisch abgrenzen lassen. ${ }^{35}$

Eine vorsichtige Relativierung bezieht sich auf die bisherige Anforderung, wonach die Messbarkeit von Zusatzeinträgen ausschlaggebend sei. Diese sei, so der 9. Senat, nicht allein maßgebend, es komme vielmehr auf die Möglichkeit der Zuordnung der Stickstoffdeposition zu einer bestimmten Quelle an. ${ }^{36}$

Die Begründungen der Rechtsprechung für einen absoluten Abschneidewert in Höhe von $0,3 \mathrm{~kg} \mathrm{~N} /\left(\right.$ ha ${ }^{\star}$ a) können also wie folgt zusammengefasst werden:

(1) Einträge unter $0,3 \mathrm{~kg} \mathrm{~N} / \mathrm{ha} \star \mathrm{a}$ ) liegen unterhalb der Grenze messtechnischer Nachweisbarkeit und können einem Emittenten bzw. einer Quelle nicht zugeordnet werden. Damit fehlt es am Nachweis der kausalen Zusammenhänge zwischen Emission und Deposition, der von $\$ 34$ BNatSchG aber gefordert wird.

(2) Unterhalb von $0,3 \mathrm{~kg} \mathrm{~N} /($ ha $a$ ) ist die zusätzliche von einem Vorhaben ausgehende Belastung nicht mehr

22) Zum Ganzen instruktiv Gellermann, Projektbedingte Stickstoffbelastungen von Natura-2000-Gebieten, NuR 2019, S. $747 \mathrm{ff}$.

23) Balla et al., „Untersuchung und Bewertung von straßenverkehrsbedingten Nährstoffeinträgen in empfindliche Biotope", Bericht zum FE-Vorhaben 84.0102/2009 der Bundesanstalt für StraBenwesen, Forschung Straßenbau und Straßenverkehrstechnik Bd. 1099, hrsg. vom Bundesministerium für Verkehr, Bau und Stadtentwicklung - BMVBS -, November 2013; im Folgenden „FE-Bericht Stickstoff 2013“ (Bezeichnung aus BVerwG, Urt. v. 23. 4.2014 - 9 A 25.12, NuR 2014, 706, Rdnr. 37).

24) FGSV, Hinweise zur Prüfung von Stickstoffeinträgen in der FFH-Verträglichkeitsprüfung für Straßen - H PSE, Endfassung 2019, im Folgenden ,Stickstoffleitfaden Straße 2019“.

25) Balla et al., ,,Stickstoffeinträge in der FFH-Verträglichkeitsprüfung: Critical Loads, Bagatellschwelle und Abschneidekriterium“, in: Waldökologie, Landschaftsforschung und Naturschutz 14 (2014), S. 7 - künftig: „Balla et al., Stickstoffeinträge in der FFH-VP 2014“.

26) BVerwG, Urt. v. 23.4.2014 - 9 A 25.12, NuR 2014, 706, Rdnr. 45, mit Verweis auf den FE-Bericht Stickstoff 2013, S. $216 \mathrm{ff}$.

27) BVerwG, Urt. v. 23.4.2014 - 9 A 25.12, NuR 2014, 706 , Rdnr. 45, mit Verweis auf Balla et al., Stickstoffeinträge in der FFH-VP 2014, S. 7.

28) BVerwG, Urt. v. 23.4.2014 - 9 A 25.12, NuR 2014, 706, Rdnr. 45, mit Verweis auf FE-Bericht Stickstoff 2013, S. 217, und auf Balla et al., Stickstoffeinträge in der FFH-VP 2014, S. 6.

29) OVG Münster, Urt. v. 16.6.2016 - 8 D 99/13.AK, NuR 2017, 482, Rdnr. 602.

30) BVerwG, Urt. v. 15.5.2019 - 7 C 27.17, NuR 2019, 763 , Rdnr. $36 \mathrm{ff}$.

31) EuGH, Urt. v. 7. 11.2018 - C-293/17, NuR 2018, 852, Rdnr. 104 , 112.

32) BVerwG, Urt. v. 27.11.2018 - 9 A 8.17, NuR 2019, 608 , Rdnr. $81 \mathrm{f}$.

33) BVerwG, Urt. v. 12.6.2019 - 9 A 2.18, BVerwGE 166, 1; zur weiteren Bestätigung des Abschneidewerts, allerdings ohne neue oder andere Begründungsansätze, siehe BVerwG, Urt. v. 11.7.2019 - 9 A 13.18, NuR 2020, 253, Rdnr. 103 ff.; BVerwG, Urt. v. 3.11.2020 - 9 A 12.19, Rdnr. 422, juris.

34) BVerwG, Urt. v. 12.6.2019 - 9 A 2.18, Leitsatz 2 und Rdnr. 63 f.

35) BVerwG, Urt. v. 12.6.2019 - 9 A 2.18, Rdnr. 70, juris.

36) BVerwG, Urt. v. 12.6.2019 - 9 A 2.18, Rdnr. 71, juris. 
eindeutig von der vorhandenen Hintergrundbelastung abgrenzbar.

(3) Empirische Untersuchungen ergaben keine signifikanten schädlichen Effekte von stickstoffhaltigen Immissionen einer Straße jenseits der 3\%-Belastungsgrenze. ${ }^{37}$

(4) Einträge bis $0,3 \mathrm{~kg} \mathrm{~N} /\left(\mathrm{ha}^{\star}\right.$ a) liegen unterhalb einer sicheren Ursache-Wirkungs-Relation, weil die meisten experimentellen wissenschaftlichen Studien zu den Einflüssen zusätzlicher Stickstoffeinträge auf die Vegetation mit Stickstoffabgaben in Stufen von mindestens 5 bis $10 \mathrm{~kg} \mathrm{~N} /(\mathrm{ha} \star \mathrm{a})$ arbeiteten.

Es wird weiter unten (6.) gezeigt, dass diese Begründungsansätze weder im Hinblick auf die messtechnischen bzw. physikalischen noch auf die vegetationsökologischen Ansätze stichhaltig sind. Daraus folgt außerdem, dass es sich bei den in Bezug genommenen Forschungsberichten/Leitfäden nicht um Fachkonventionen nach den Maßstäben der Rechtsprechung handelt.

\section{Unionsrechtlicher Maßstab für den Nachweis eines Ursachenzusammenhangs}

Nach Auffassung des 9. Senats steht seine Rechtsprechung zum Abschneidekriterium nicht im Widerspruch zu einem Urteil des EuGH vom 14.1.2016. ${ }^{38}$ Der EuGH hatte darin klargestellt, dass es für einen Verstoß gegen Art. 6 Abs. 2 der FFH-Richtlinie ausreichen kann, dass die Wahrscheinlichkeit oder die Gefahr einer erheblichen Störung bestehe, ohne dass ein ursächlicher Zusammenhang nachgewiesen werden müsste. Das BVerw $\mathrm{G}^{39}$ argumentiert, dass kein Widerspruch zum EuGH bestehe, „weil das Abschneidekriterium gerade den Bereich bezeichnet, für den sich keine gewisse Wahrscheinlichkeit der Beeinträchtigung begründen lässt."

Das BVerwG verlangt in seiner Argumentation den Nachweis, dass Stickstoffeinträge messbar und einer Quelle zuordenbar sein müssen. ${ }^{40}$ Der EuGH stellt fest, dass ein ursächlicher Zusammenhang für eine erhebliche Störung nicht nachgewiesen werden müsse. Die Feststellung des EuGH bezieht sich auf den (nicht erforderlichen) Ursachenzusammenhang in der gesamten Wirkkette.

Auf der physikalisch-messtechnischen Seite reicht es somit - nach dem Ansatz des EuGH - aus, wenn es wahrscheinlich ist, dass ein Stickstoffeintrag einem bestimmten Emittenten zugeordnet werden kann. Für die Feststellung dieser Wahrscheinlichkeit ist die prognostische Ermittlung der Einträge aus einer Quelle ausreichend. Der EuGH verlangt eben gerade keinen Nachweis durch Messung. ${ }^{41}$

Auf der vegetationsökologischen Seite reicht es - nach dem Ansatz des EuGH - aus, dass die Gefahr einer erheblichen Störung durch die Stickstoffeinträge besteht. Diese Gefahr besteht nach dem habitatschutzrechtlichen Beweismaßstab, wenn vernünftige Zweifel am Ausbleiben der Gefahr vorliegen. ${ }^{42}$ Nur dann darf die Verträglichkeitsprüfung mit einem positiven Ergebnis abgeschlossen werden. ${ }^{43}$ Es wird unten (6.) gezeigt, dass in den als Fachkonvention angesehenen Untersuchungen (Forschungsbericht Stickstoff 2013, Stickstoffleitfaden Straße 2019) kein Nachweis über das Vorliegen empirischer Untersuchungen geführt wird, die bei Einträgen bis $0,3 \mathrm{~kg} \mathrm{~N} /\left(\mathrm{ha}{ }^{\star} \mathrm{a}\right)$ die Entstehung von Beeinträchtigungen ausschließen. Es wird weiter gezeigt, dass Einträge bis $0,3 \mathrm{~kg} \mathrm{~N} /\left(\mathrm{ha}^{\star} \mathrm{a}\right)$ schädigende Wirkung haben können.

Die Frage, welche Anforderungen an die Wahrscheinlichkeit oder Gefahr einer erheblichen Störung zu stellen sind, ist in der Rechtsprechung des BVerwG außerdem dogmatisch nicht eindeutig verortet. Der 7. Senat urteilte hierzu im Rahmen einer Revision ${ }^{44}$ und klärte somit eine Rechtsfrage, während der 9. Senat (als erstinstanzliches Tatsachengericht) der Tatsachenfrage nachging, ob die Wahrscheinlichkeit oder Gefahr einer erheblichen Störung durch Einträge unterhalb $0,3 \mathrm{~kg} \mathrm{~N} /\left(\mathrm{ha}^{\star} \mathrm{a}\right)$ besteht. ${ }^{45}$
Da die Beantwortung der Tatsachenfrage vom rechtlichen Maßstab abhängt, handelt es sich in erster Linie um eine Rechtsfrage, für deren Klärung der EuGH zuständig ist. Die unterschiedliche Einordnung des Abschneidewerts als Rechtsfrage (7. Senat) oder als Tatsachenfrage (9. Senat) hätte außerdem die Befassung des Großen Senats des BVerwG erfordert ( $\$ 11 \mathrm{VwGO})$.

\section{Der Abschneidewert als Fachkonvention}

Der Forschungsbericht Stickstoff 2013 enthält einen Fachkonventionsvorschlag zur Erheblichkeitsbeurteilung für Stickstoffeinträge. ${ }^{46}$ Der Stickstoffleitfaden Straße 2019 qualifiziert sich - auf der Grundlage des Forschungsberichts 2013 - selbst als Fachkonvention. ${ }^{47}$ Die Rechtsprechung folgt dem und attestiert sowohl dem Forschungsbericht 2013 als auch dem Stickstoffleitfaden 2019 den Status einer Fachkonvention $^{48}$, die den aktuell besten wissenschaftlichen Erkenntnisstand widerspiegelt. ${ }^{49}$ Anhaltspunkte für bessere wissenschaftliche Erkenntnisse, die geeignet wären, Methodik, Grundannahmen oder Schlussfolgerungen des Stickstoffleitfadens substantiell in Frage zu stellen oder zu widerlegen, gebe es nicht, so dass die Grenzen der möglichen und gebotenen gerichtlichen Aufklärung erreicht seien. ${ }^{50}$

Voraussetzung für die Anerkennung einer Arbeit als Fachkonvention ist, dass sie die besten wissenschaftlichen Erkenntnisse widerspiegelt. Dies muss seitens der Gerichte

37) Diese Feststellung in den Leitfäden/Berichten bezieht sich nicht auf die absolute Schwelle von $0,3 \mathrm{~kg} \mathrm{~N} / \mathrm{ha}^{\star} \mathrm{a}$ ), sondern auf den schutzgutbezogenen Wert von $3 \%$ des Critical Loads. Sie kann aber für den Fall, dass der Critical Load bei $10 \mathrm{~kg} \mathrm{~N} /\left(\right.$ ha $\left.{ }^{\star} \mathrm{a}\right)$ liegt, herangezogen werden, weil 3\% dann der Schwelle von $0,3 \mathrm{~kg}$ N/ (ha $\left.{ }^{\star} \mathrm{a}\right)$ entsprechen

38) EuGH, Urt. v. 14.1.2016 - C-399/14, ECLI:EU:C:2016:10, Rdnr. 42.

39) BVerwG, Urt. v. 12.6.2019 - 9 A 2.18, Rdnr. 72, juris.

40) Siehe hierzu OVG Münster, Urt. v. 16.6.2016 - 8 D 99/13.AK, NuR 2017, 482, Rdnr. 558: ,empirisch nachweisbare Wirkungsschwellen“" (unter Bezugnahme auf den Forschungsbericht Stickstoff 2013); BVerwG, Urt. v. 15. 5.2019 - 7 C 27.17, NuR 2019, 763, Rdnr. 37: ,empirisch aber weder nachweisbarer noch wirkseitig zuordenbarer Eintragswert"; BVerwG, Urt. v. 12.6.20199 A 2.18, Rdnr. 70, juris: „Der vorhabenbedingte Eintrag muss nicht nur messtechnisch nachweisbar sein ...; Rdnr. 71, juris: „Bei Depositionsraten, die bei $0,3 \mathrm{~kg} \mathrm{~N} / \mathrm{ha} / \mathrm{a}$ oder darunter liegen, lässt sich kein kausaler Zusammenhang zwischen Emission und Deposition herstellen, der Eintrag liegt unterhalb nachweisbarer Wirkungen auf die Schutzgüter der FFH-Richtlinie ..." (unter Bezugnahme auf den Stickstoffleitfaden Straße).

41) Es wird weiter unten außerdem gezeigt, dass sowohl die Messbarkeit von Einträgen unter $0,3 \mathrm{~kg} \mathrm{~N} /\left(\mathrm{ha}^{\star} \mathrm{a}\right)$ als auch deren $\mathrm{Zu}$ ordenbarkeit als auch deren Unterscheidbarkeit von der Hintergrundbelastung technisch möglich sind.

42) EuGH, Urt. v. 7. 9. 2004 - C-127/02, Slg. 2004, I-7405, Rdnr. 59 und 61; BVerwG, Urt. v. 17.1.2007 - 9 A 20.05, BVerwGE 128 , 1 Rdnr. 56; BVerwG, Urt. v. 11.8.2016 - 7 A 1.15, BVerwGE 156, 20 Rdnr. 67.

43) BVerwG, Urt. v. 6.4.2017 - 4 A 16.16, Rdnr. 33, juris.

44) BVerwG, Urt. v. 15. 5.2019 - 7 C 27.17, NuR 2019, 763

45) Der 9. Senat (BVerwG, Urt. v. 12.6.2019-9 A 2.18, Rdnr. 63f, juris) nahm hierzu die als Fachkonventionen eingeordneten Untersuchungen im Forschungsbericht 2013 und im Stickstoffleitfaden 2019 in Bezug; da eine Fachkonvention keine Rechtsfrage klären kann, kann es sich also nur um die Klärung einer Tatsachenfrage handeln.

46) FE-Bericht Stickstoff 2013, Bild 76, S. 212

47) Stickstoffleitfaden Straße 2019, S. 1

48) BVerwG, Urt. v. 27.11.2018 - 9 A 8.17, NuR 2019, 608 , Rdnr. 79; BVerwG, Urt. v. 15. 5.2019 - 7 C 27.17, NuR 2019, 763 , Rdnr. 37; BVerwG, Urt. v. 12.6.2019 - 9 A 2.18, Rdnr. 63 ff., juris; BVerwG, Urt. v. 21.1.2021 - 7 C 9.19, Rdnr. 23, juris.

49) BVerwG, Urt. v. 12.6.2019 - 9 A 2.18, Rdnr. 63 ff., juris; BVerwG, Urt. v. 21.1.2021 - 7 C 9.19, Rdnr. 23, juris.

50) BVerwG, Urt. v. 12.6.2019 - 9 A 2.18, Rdnr. 64, juris. 
überprüft werden. ${ }^{51}$ Eine solche Überprüfung ist immer dann erforderlich, wenn zentrale Begründungsansätze einer Fachkonvention durch Verweis auf entgegenstehende und dokumentierte Erkenntnisse hinterfragt werden.

In einer solchen Konstellation geht es auch nicht um die Ersetzung einer Bewertung durch eine andere Bewertung, sondern um die Überprüfung der Richtigkeit naturwissenschaftlicher Aussagen als Grundlage einer daraus - in einer Fachkonvention - abgeleiteten Bewertung. Sind naturwissenschaftliche Aussagen überprüfbar und werden sie substantiiert in Zweifel gestellt, müssen sie von den Gerichten überprüft werden. Das BVerfG ${ }^{52}$ verlangt im Hinblick auf entscheidungserhebliche Tatsachen die weitestmögliche Aufklärung (Leitsatz 1 und Rdnr. 25), die vollständige Nachprüfung in rechtlicher und tatsächlicher Hinsicht (Rdnr. 19) und die weitestgehend mögliche Kontrolle der Behördenentscheidung (Rdnr. 25). Auch der EuGH verlangt im Habitatschutzrecht in ständiger Rechtsprechung die Anwendung der besten wissenschaftlichen Erkenntnisse ${ }^{53}$. Die Zugrundelegung einer Fachkonvention, deren tatsächliche Grundlagen nicht stimmen, würde einen Verstoß gegen Unionsrecht nach sich ziehen.

Die TA Luft hat in ihrer am 1.12.2021 in Kraft tretenden Neufassung ${ }^{54}$ das Abschneidekriterium von $0,3 \mathrm{~kg} \mathrm{~N} /$ $\left(\mathrm{ha}^{\star} \star_{\mathrm{a}}\right)$ übernommen. ${ }^{55}$

Die Übernahme in die TA Luft verleiht dem Abschneidewert keine höhere Verbindlichkeit. Die TA Luft ist diesbezüglich bereits wegen des unionsrechtlichen Verstoßes unanwendbar. ${ }^{56}$

Dass es sich bei der TA Luft nicht um eine die gesetzlichen Vorgaben in zulässiger Weise konkretisierende Verwaltungsvorschrift handelt, sondern einen politischen Kompromiss, zeigt sich im Übrigen auch in deren Anhang 9. Darin wird für die Stickstoffdeposition (außerhalb von FFH-Gebieten) eine Bagatellgrenze von $5 \mathrm{~kg} \mathrm{~N}$ (ha ${ }^{\star}$ ) festgelegt. Diese dem Leitfaden zur Ermittlung und Bewertung von Stickstoffeinträgen der Bund/Länder-Arbeitsgemeinschaft für Immissionsschutz, Stand März 2021 entnommene Grenze hat das BVerw $\mathrm{G}^{57}$ aus materiellen Gründen als rechtswidrig qualifiziert, weil die Schwelle viel zu hoch ist (Rdnr. 31: „Eine derart hohe Zusatzbelastung überschreitet das Bagatellhafte deutlich."). Die TA Luft konkretisiert also nicht die gesetzliche Vorgabe, sie ist daher keine zulässige Normkonkretisierung.

Der Forschungsbericht Stickstoff 2013 und der Stickstoffleitfaden Straße 2019 erfüllen demnach nicht (mehr) die Anforderungen an eine Fachkonvention im Hinblick auf das Abschneidekriterium. Die Rechtsprechung wird die gegen die Begründungsansätze des Abschneidekriteriums vorgebrachten physikalisch-technischen und vegetationsökologischen Argumente überprüfen müssen, wenn es zur Urteilsfindung auf das Abschneidekriterium ankommt.

\section{Fehlerhafte Herleitung des Abschneidewerts in technisch-mathematischer und vegetationsökologischer Hinsicht}

Die Rechtsprechung beruft sich auf verschiedene Begründungen des Abschneidewerts sowohl in technisch-mathematischer Hinsicht (Messuntergrenze, Zuordenbarkeit zu einer Quelle, statistische Unterscheidbarkeit von der Hintergrundbelastung) als auch in vegetationsökologischer Sicht (untere Wirkschwelle, fehlende Ursache-WirkungsRelation). Diese Begründungansätze werden im wesentlichen drei Arbeiten entnommen. ${ }^{58}$ Im Folgenden wird aufgezeigt, dass diese Begründungsansätze falsch bzw. nicht nachvollziehbar hergeleitet sind und daher der Abschneidewert nicht verwendet werden kann.

\section{1 technisch-mathematische Herleitung des Abschneidewerts ${ }^{59}$}

Im FE-Bericht Stickstoff 2013 wird der Abschneidewert von $0,3 \mathrm{~kg} \mathrm{~N} /(\mathrm{ha}$ a) damit begründet, dass dies die (hal- bierte) untere Grenze der messtechnischen Nachweisbarkeit $\operatorname{sei}^{60}$. Tatsächlich liegt (und lag auch 2013) die Nachweisbarkeit wesentlich niedriger.

Der FE-Bericht Stickstoff aus 2013 leitet den Abschneidewert entscheidend aus der technischen Messbarkeit der Konzentration und anschließender Umrechnung in die Deposition ab. Begründet wird dies mit der unkomplizierteren Messbarkeit von Konzentrationen. ${ }^{61}$ Die Angabe als Depositionswert erfolgt im FE-Bericht als aus der Konzentration berechnete Größe mit den Umrechnungsfaktoren für die trockene Deposition gemäß VDI-Richtlinie 3782 Blatt 5.

Die unteren Nachweisgrenzen ${ }^{62}$ für die Konzentrationen von Stickstoffverbindungen werden im FE-Bericht Stickstoff 2013 jedoch nicht korrekt ermittelt. Die wenigen Angaben zu den betrachteten Geräten finden sich lediglich in einer informationsarmen Fußnote. ${ }^{63}$ Eine vollständige Auflistung oder die Auswahlkriterien für diese ,ca. 20 Messgeräte" erfolgt nicht. Es ist erstaunlich, dass die wesentliche Angabe konkreter Messgeräte, aufgrund deren Eigenschaf-

51) Bick/Wulfert, Der Artenschutz in der Vorhabenzulassung aus rechtlicher und naturschutzfachlicher Sicht, NVwZ 2017, S. $346 \mathrm{ff}$., verweisen auf die Anforderungen des EuGH zur Zugrundelegung der besten wissenschaftlichen Erkenntnisse und leiten daraus im Hinblick auf Fachkonventionen eine gerichtliche Pflicht zur Plausibilitätsprüfung und der Übereinstimmung mit den rechtlichen Vorgaben ab.

52) BVerfG, Beschl. v. 23.10.2018 - 1 BvR 2523/13, NuR 2019, 33

53) EuGH, Urt. v. 7. $9.2004-$ C-127/02, Slg. 2004, I-7405, Rdnr. 54 EuGH, Urt. v. 23.4.2020 - C-217/19, ECLI:EU:C:2020:291, Rdnr. 70, ständige Rechtsprechung.

54) Gemeinsames Ministerialblatt, Ausgabe 48-54/2021, S. $1050 \mathrm{ff}$.

55) Ziffer 4.8 „Prüfung der Verträglichkeit von Stickstoff- und Säureeinträgen für Gebiete von gemeinschaftlicher Bedeutung“", mit Verweis auf Anhang 8.

56) BVerwG, Urt. v. 27.2. 2018 - 7 C 30.17, Rdnr. 36, juris; EuGH, Urt. v. 20.12.2017 - C-664/15, NuR 2018, 395, Rdnr. 55.

57) BVerwG, Urt. v. 21.1.2021- 7 C 9.19, juris Rdnr. 30 f.

58) FE-Bericht Stickktoff 2013, Stickstoffleitfaden Straße 2019, Balla et al.; Stickstoffeinträge in der FFH-VP 2014, siehe oben Fn. 22-24.

59) Grundlage dieses Textteils ist die Arbeit „Untersuchung des Abschneidekriteriums von $0,3 \mathrm{~kg} \mathrm{~N} /\left(\mathrm{ha} \star^{\star}\right.$ ) aus technisch-mathematischer Perspektive“, Dr. Thomas Krämerkämper, Mai 2021, die im gerichtlichen Verfahren BVerwG 9 A 1.21 vorgelegt wurde.

60) FE-Bericht Stickstoff 2013, S. 93 f.; siehe auch BVerwG, Urt. v. 12.6.2019 - 9 A 2.18, Rdnr. 70.

61) Dieses Vorgehen ist nicht nur aufgrund der einfacheren Messbarkeit der Konzentrationen sinnvoll. Luftschadstoffe wirken nicht erst über die Deposition, also den Eintrag auf Oberflächen, sondern bereits durch ihre Konzentration in der Umgebungsluft. Analog zu den Critical Loads für die Deposition werden für Ökosysteme die Effektschwellen der Konzentration von Luftschadstoffen als Critical Level angegeben. Zur Definition siehe Richtlinie 2001/81/EG des Europäischen Parlaments und des Rates vom 23. Oktober 2001 über nationale Emissionshöchstmengen für bestimmte Luftschadstoffe, Amtsblatt Nr. L 309 vom 27.11.2001 S. 0022-0030, Art. 3 lit. d): „kritische Konzentration“ die Konzentration von Schadstoffen in der Luft, bei deren Überschreitung nach dem gegenwärtigen Erkenntnisstand direkte negative Folgen für Rezeptoren, wie Menschen, Pflanzen, Ökosysteme oder Materialien, eintreten können. Auch aus diesem Grund und dem zusätzlichen Wirkpfad ist eine Definition von Abschneidewerten als Konzentration anstelle der Deposition sinnvoll.

62) Angesichts der hohen bodennahen Hintergrundbelastung in Deutschland wird niemals an der unteren Nachweisgrenze gemessen. Relevanter wäre vielmehr die Genauigkeit von Messungen bei den vorliegenden Hintergrundkonzentrationen.

63) FE-Bericht Stickstoff 2013, S. 94: „Es wurden ca. 20 Messgeräte für die Konzentration von Außenluft und Abgasluft ausgewertet. Niedrigste Nachweisgrenze für $0,4 \mu \mathrm{g} \mathrm{NO}_{\mathrm{x}} / \mathrm{m}^{3}$ : Thermo-Analysator Modell TE42C (www.umweltbundesamt.de/luft/messeinrichtungen/5Anhang3.pdf;. Niedrigste Nachweisgrenze für 0,14 $\mu \mathrm{g} \mathrm{NH}_{3} / \mathrm{m}^{3}$ : Prometh (Schlupfmessung in Prozessabgasen) MI-PAN. (http://www.prometh.de/html/applikationsbericht.html)“. 
Tabelle 1 Messungen bzw. Messgeräte für Stickoxide

\begin{tabular}{lllc}
\hline & \multicolumn{2}{l}{ untere Nachweisgrenze } & \\
Methode bzw. Gerät & NO - NO & Jahr \\
\hline [Volz et al. 1984] $^{64}$ & $5-10 \mathrm{ppt}$ & $0,006-0,019 \mu \mathrm{g} / \mathrm{m}^{3}$ & 1983 \\
Ecophysics CLD780TR & $3 \mathrm{ppt}$ & $0,004-0,006 \mu \mathrm{g} / \mathrm{m}^{3}$ & $\mathrm{ca} .1996$ \\
Ecophysics CLD790SR & $1,5-2,2 \mathrm{ppt}$ & $0,002-0,004 \mu \mathrm{g} / \mathrm{m}^{3}$ & 2011 \\
QUMA LOPAP- & $2 \mathrm{ppt}$ & $0,004 \mu \mathrm{g} / \mathrm{m}^{3}$ & 2012 \\
03-NO2 (nur NO & \\
FE-Bericht Stickstoff 2013 & $209-321 \mathrm{ppt}$ & $0,4 \mu \mathrm{g} / \mathrm{m}^{3}$ & 2013
\end{tabular}

Tabelle 2 Messgeräte für Ammoniak

\begin{tabular}{llcc}
\hline Methode bzw. Gerät & \multicolumn{1}{c}{ untere Nachweisgrenze $\mathbf{N H}_{3}$} & Jahr \\
\hline Omnisens TGA310 & $100 \mathrm{ppt}$ & $0,071 \mu \mathrm{g} / \mathrm{m}^{3}$ & 2004 \\
Aerodyne QC-TILDAS & $42 \mathrm{ppt}$ & $0,030 \mu \mathrm{g} / \mathrm{m}^{3}$ & 2010 \\
Minidenuder & $28 \mathrm{ppt}$ & $0,02 \mu \mathrm{g} / \mathrm{m}^{3}$ & 2012 \\
Umweltbundesamt & & & \\
Picarro G2103 & & $0,014 \mu \mathrm{g} / \mathrm{m}^{3}$ & 2012 \\
Aerodyne Mini-TILDAS & $21 \mathrm{ppt}$ & $0,015 \mu \mathrm{g} / \mathrm{m}^{3}$ & 2020 \\
FE-Bericht Stickstoff 2013 & $198 \mathrm{ppt}$ & $0,14 \mu \mathrm{g} / \mathrm{m}^{3}$ & 2013 \\
\hline
\end{tabular}

ten die Herleitung des Abschneidekriteriums erfolgt, sowohl im FE-Bericht als auch in allen einschlägigen Folgeveröffentlichungen unterblieben ist. Inhaltlich stellt sich diese Marktanalyse von Messgeräten zudem als irreführend heraus. Eine Recherche der Autoren unter einschlägigen Herstellern von Messgeräten für den Feldeinsatz ergibt ohne Anspruch auf Vollständigkeit - das in Tabelle 1 und Tabelle 2 dargestellte Bild ${ }^{67,68}$.

Entgegen der Darstellung im FE-Bericht Stickstoff 2013 lag also die untere Nachweisgrenze von Stickoxiden schon 30 Jahre zuvor um zwei Größenordnungen niedriger als in dem Bericht angesetzt. Für Ammoniak liegt und lag schon 2013 die untere Nachweisgrenze um eine Größenordnung niedriger, als der FE-Bericht Stickstoff 2013 behauptet. Offensichtlich fehlt es im FE-Bericht Stickstoff 2013 an einer wissenschaftlichen Ermittlung der unteren Nachweisgrenzen.

Die beiden im FE-Bericht Stickstoff 2013 gemäß der Fußnote 83 auf S. 94 behaupteten ,,besten Messgeräte“ sind nicht auf die Messung möglichst niedrigerer Konzentrationen in der Umgebungsluft spezialisiert. Sie sind vielmehr für die Messung im Abgasstrom ausgelegt, also zur Emissionskontrolle anstatt zur Immissionskontrolle. Besonders auffällig ist dies bei der ungeeigneten Auswahl des vermeintlich besten Messgeräts für die Messung von Stickoxiden: Der FE-Bericht Stickstoff 2013 behauptet, das Gerät Thermo Electron TE42C sei das beste Gerät mit der niedrigsten unteren Nachweisgrenze für $\mathrm{NO}_{\mathrm{X}}$ von $0,4 \mu \mathrm{g} / \mathrm{m}^{3}$. Jedoch wird diese Gerätevariante vom Hersteller seit 2004 speziell für die Emissionskontrolle produziert. Für die Spurengasanalyse in der Umgebungsluft sah derselbe Hersteller bereits seit 2003 die genauere Modellvariante Thermo Electron TE42C-TL vor (heutige Bezeichnung TE42i-TL; das „TL“ steht für Trace Level, d.h. Spurengaskonzentrationen). Für diese Variante gibt der Hersteller eine wesentlich niedrigere untere Nachweisgrenze von $0,1 \mu \mathrm{g} / \mathrm{m}^{3}$ für $\mathrm{NO}$ und $0,15 \mu \mathrm{g} / \mathrm{m}^{3}$ für $\mathrm{NO}_{2}$ an. Da die Messgeräte anderer Hersteller noch wesentlich genauer sind, ist diese TL-Variante jedoch in der oben dargestellten Tabelle nicht aufgeführt.

Mit den hier aufgelisteten Messgeräten kann die ansonsten gleiche Berechnungsmethodik wie im FE-Bericht Stickstoff 2013 angewendet werden. Damit hätten sich be-
Tabelle 3 resultierende Abschneidewerte nach dem Berechnungsverfahren aus dem FE-Bericht Stickstoff 2013

\begin{tabular}{lcc}
\hline Stoff & $\begin{array}{c}\text { Abschneide- } \\
\text { kriterium }\end{array}$ & $\begin{array}{c}\text { Depositionsäquivalent für die } \\
\text { trockene Deposition, Mesoskala }\end{array}$ \\
\hline $\mathrm{NO}$ & $0,0019 \mu \mathrm{g} / \mathrm{m}^{3}$ & $0,0001 \mathrm{~kg} \mathrm{~N} /\left(\mathrm{ha} \star_{\mathrm{a}}\right)$ \\
$\mathrm{NO}_{2}$ & $0,0019 \mu \mathrm{g} / \mathrm{m}^{3}$ & $0,0006 \mathrm{~kg} \mathrm{~N} /\left(\mathrm{ha} \star_{\mathrm{a}}\right)$ \\
$\mathrm{NH}_{3}$ & $0,0074 \mu \mathrm{g} / \mathrm{m}^{3}$ & $0,0231 \mathrm{~kg} \mathrm{~N} /\left(\mathrm{ha} \star_{\mathrm{a}}\right)$ \\
\hline
\end{tabular}

reits im Jahr 2013 die in Tabelle 3 aufgelisteten Abschneidewerte ergeben müssen.

In Summe wären das also $0,024 \mathrm{~kg} \mathrm{~N} /\left(\mathrm{ha}^{\star} \mathrm{a}\right)$ anstelle der im FE-Bericht Stickstoff 2013 postulierten $0,3 \mathrm{~kg} \mathrm{~N} /\left(\mathrm{ha}^{\star} \mathrm{a}\right)$. Inzwischen liegen die unteren Nachweisgrenzen im wissenschaftlichen Bereich sogar noch deutlich niedriger im Sub-ppt-Bereich.

Die Abschneidewerte müssen außerdem stoffspezifisch angesetzt werden, da die zugrunde liegende Messtechnik stoffspezifisch ist. Insbesondere gibt es keinen wissenschaftlichen Grund, warum die Immissionen einer Quelle, die nur Stickoxide emittiert, aufgrund der höheren unteren Nachweisgrenze von Ammoniak zwei Größenordnungen höher abgeschnitten werden sollte, als es sich aus der Messtechnik für Stickoxide ergibt.

Neben der ungeeignet gewählten Messtechnik wird im FE-Bericht Stickstoff 2013 die Wirkung der unteren Nachweisgrenze zudem künstlich verschlechtert, indem auf den Jahresmittelwert der Konzentration abgestellt wird, anstatt auf ein realistisches Messintervall. Die meisten o. g. Messgeräte erreichen ihre unteren Nachweisgrenzen typischerweise bei Messintervallen von nur wenigen Minuten. Wird nun ein aus diesen unteren Nachweisgrenzen abgeleiteter Abschneidewert nicht mehr auf die korrespondierenden Messintervalle der Messgeräte angewendet, sondern nur auf den Jahresmittelwert, so werden die prognostizierten, an einem konkreten Beurteilungspunkt beitragenden Immissionen eines Vorhabens zeitlich ,verdünnt". Denn an einem konkreten Beurteilungspunkt tragen typischerweise nicht mehr als 50 \% der Emissionsstunden zu den Immissionen eines Vorhabens bei, selbst wenn dieser Beurteilungspunkt in der Hauptwindrichtung zur Quelle liegt. In den restlichen Emissionsstunden bläst der Wind in Richtungen, bei denen die Abgasfahne den Beurteilungspunkt nicht erreichen kann. Bei den meisten Beurteilungspunkten außerhalb der Hauptwindrichtung sind es sogar deutlich weniger als 50\% der Emissionsstunden, an denen Immissionen durch das Vorhaben erfolgen können. Durch die Jahresmittelwertbildung muss also zu den tatsächlich beitragenden Emissionsstunden ei-

64) Volz und Drummond, ,Messung von Stickoxiden im ppt-Bereich durch Chemiluminescenz mit O3“, Fresenius Zeitschrift für Analytische Chemie (1984) 317:355-358 Springer Verlag.

65) Modifizierte Version, s. Hosaynali Beygi/Fischer/Harder/Martinez/ Sander/Williams/Brookes/Monks and Lelieveld: „Oxidation photochemistry in the Southern Atlantic boundary layer: unexpected deviations of photochemical steady state", Atmos. Chem. Phys., 11, 8497-8513, https://doi.org/10.5194/acp-11-8497-2011, 2011.

66) Die Angabe erfolgt für dieses Modell als „,typischer“ Wert, der für mindestens die Hälfte der produzierten Geräte erreicht wird.

67) Die Umrechnung zwischen den beiden Konzentrationseinheiten ppt und $\mu \mathrm{g} / \mathrm{m}^{3}$ erfolgt hier jeweils für $20^{\circ} \mathrm{C}$ und 1013,25 mbar.

68) Die untere Nachweisgrenze wird von vielen Herstellern im 2-Sigma- oder 3-Sigma-Standard angegeben und hier so übernommen. Demgegenüber scheint im FE-Bericht Stickstoff 2013 lediglich ein 1-Sigma verwendet zu sein, wie sich aus dem Vergleich der Angabe zum TE42C im Bericht und im Datenblatt des Herstellers ergibt. Würde auf die hier genannten Geräte ebenso lediglich ein 1-Sigma-Standard angewendet, ergäbe sich ein nochmals deutlich niedrigerer Abschneidewert. 
nes Vorhabens die Konzentration mindestens beim Doppelten der unteren Nachweisgrenze liegen, um im Jahresmittelwert über alle Emissionsstunden über die untere Nachweisgrenze zu kommen. Daher ist die Betrachtung des Jahresmittelwertes methodisch ungeeignet. Stattdessen wären die Abschneidewerte auf tatsächliche realistische Messintervalle anzuwenden, hilfsweise auf das typische Prognoseintervall einer Stunde.

Weder die Messgeräteauswahl noch die Methodik im FE-Bericht Stickstoff 2013 werden somit dem Anspruch einer möglichst optimalen Ermittlung gerecht. Der resultierende Abschneidewert im FE-Bericht Stickstoff 2013 ist daher quantitativ keine wissenschaftlich gestützte Größe. Es fehlt außerdem an der Erfüllung der gebotenen wissenschaftlichen Dokumentations- und Transparenzpflicht bzgl. der Messgeräte, um den FE-Bericht Stickstoff 2013 diesbezüglich als wissenschaftliche Arbeit bezeichnen zu können.

In der von der Rechtsprechung herangezogenen Veröffentlichung ,Balla et al., Stickstoffeinträge in der FFH-VP 2014" 69 aus dem Jahr 2014 soll derselbe Abschneidewert wie im FE-Bericht Stickstoff 2013 von $0,3 \mathrm{~kg} \mathrm{~N} /\left(\mathrm{ha}^{\star} \mathrm{a}\right)$ durch eine völlig andere Herleitung gerechtfertigt werden. Diese Herleitung behauptet im Wesentlichen, dass bei kleineren Zusatzbelastungen diese nicht mehr von zufälligen Variationen der Hintergrundbelastung zu unterscheiden wäre. Als $\mathrm{Maß}$ für die zufälligen Variationen der Hintergrundbelastung wird in dem Aufsatz die Unsicherheit der für die Simulationen verwendeten Computermodelle verwendet. Auch diese Herleitung ist wissenschaftlich aus mehreren Gründen nicht haltbar

Zunächst wird schon die verwendete Modellunsicherheit falsch interpretiert. Eine wissenschaftliche Unsicherheit besteht immer aus zwei Komponenten, einer systematischen und einer statistischen. Der systematische Anteil ist kein Maß für die zufälligen Schwankungen der Belastungen. Er beschreibt vielmehr eine systematische, d.h. definitionsgemäß nicht zufällige Abweichung eines Modellergebnisses vom realen Wert.

Zufällige Variationen - wie in der Veröffentlichung „Balla et al., Stickstoffeinträge in der FFH-VP 2014“ thematisiert - sind statistische Schwankungen. Die Unterschiede durch solche statistischen Schwankungen lassen sich immer beliebig reduzieren, indem die Statistik durch einen größeren simulierten oder gemessenen Stichprobenumfang verbessert wird. Dies ist eine grundlegende Erkenntnis der statistischen Mathematik. Für eine statistisch signifikante Unterscheidbarkeit zwischen einer Hintergrundbelastung und einer Gesamtbelastung gibt es in der klassischen Physik keine theoretische untere Grenze bzgl. zufälliger Schwankungen. Jede genehmigungspflichtige Emissionsquelle hat einen Effekt in Form einer zusätzlichen Konzentration bzw. Deposition. Bei einer genügend hohen Anzahl von Messungen ist ein solcher Effekt statistisch signifikant messbar, egal wie klein er ist. Jede gewünschte Genauigkeit ist für die Unterscheidbarkeit bei zufälligen Schwankungen herstellbar, indem die Stichprobenumfänge entsprechend groß gewählt werden. Dies ist ausschließlich eine Frage des Aufwands.

Zudem gilt für die Prognoseebene auf der Basis von Computermodellen $^{70}$, dass sich die Frage einer statistischen Unterscheidbarkeit von Hintergrundbelastung und Gesamtbelastung gar nicht stellt. Es ist dem beauftragten Gutachterbüro jederzeit bekannt, welcher Wert aus dem Datensatz der Hintergrundbelastung entnommen wurde und welcher Wert aus der mit einem gesonderten Simulationsprogramm in einem gesonderten Rechenlauf ermittelten Zusatzbelastung stammt. Eine statistische Unterscheidung dieser beiden Werte ist nicht erforderlich, da sie gar nicht erst vermischt werden.

Nur für eine Messung der realen Werte nach Inbetriebnahme eines Vorhabens zwecks Überprüfung der mit- tels Computermodellen simulierten Prognoseergebnisse gibt es einen Anwendungsfall für eine statistische Unterscheidung. Bei einer Messung könnte die Zusatzbelastung auch getrennt erfasst werden, falls sie chemisch oder physikalisch gekennzeichnet wird ${ }^{71}$, so dass sich der Konzentrationsbeitrag des Vorhabens bereits messtechnisch eindeutig von der Hintergrundbelastung unterscheiden lässt. Geschieht eine solche Kennzeichnung jedoch nicht, so kann die Unterscheidung nur noch statistisch erfolgen. Für eine solche statistische Unterscheidung von Messungen ist jedoch nicht, wie im Aufsatz „Balla et al., Stickstoffeinträge in der FFH-VP 2014“ behauptet, die Unsicherheit der Computermodelle relevant. Es spielt stattdessen nur die Unsicherheit der Messgeräte eine Rolle, die weitaus geringer ist als diejenige der Computermodelle. Somit lässt sich bereits bei Einzelmessungen eine weitaus bessere statistische Unterscheidbarkeit erzielen, als im Aufsatz „Balla et al., Stickstoffeinträge in der FFH-VP 2014“ dargestellt. Zudem gilt das zuvor Gesagte über die beliebige Reduzierbarkeit zufälliger Unsicherheiten durch Mehrfachmessungen. Daher kann bei solchen Mehrfachmessungen jeder noch so kleine $\mathrm{Zu}$ satzbeitrag statistisch von der Hintergrundbelastung unterschieden werden, sofern die Anzahl der Messungen nur groß genug gewählt wird.

Es lässt sich also in keinem Fall überhaupt ein Abschneidewert aus einer statistischen Unsicherheit herleiten.

Die Herleitung eines Abschneidewerts von $0,3 \mathrm{~kg} \mathrm{~N} /\left(\mathrm{ha} \star_{\mathrm{a}}\right)$ lässt sich also weder mit der Messtechnik noch einer fehlenden Unterscheidbarkeit von der Hintergrundbelastung begründen.

\subsection{Vegetationsökologische Herleitung des Abschneidewerts ${ }^{72}$}

Im FE-Bericht Stickstoff 2013 wird auf S. 218 ausgeführt, es gebe keine Anhaltspunkte, dass zusätzliche Stickstoffeinträge in der Größenordnung von $3 \%$ des für eine Pflanzengesellschaft geltenden Critical Loads (CL) für sich genommen signifikante, d.h. konkret beobachtbare Wirkungen, in der Vegetation hervorrufen könnten. Hierzu lägen empirische Studien auch außerhalb des Umfeldes von Straßen vor. Stickstoffeinträge von 0,3 bis $0,6 \mathrm{~kg} \mathrm{~N} /(\mathrm{ha}$ a) lägen deshalb unterhalb des Bereichs einer empirisch nachweisbaren Wirkungsschwelle. Zudem wird ausgeführt, dass wissenschaftliche Studien zu Einflüssen zusätzlicher Stickstoffeinträge mit Stickstoffgaben in einer Größenordnung von mindestens $5-20 \mathrm{~kg} \mathrm{~N} /\left(\mathrm{ha}^{\star} \mathrm{a}\right.$ ) arbeiteten. ${ }^{73}$ Dies wird von den Autoren des Forschungsberichts als Hinweis auf eine Wirkungsschwelle gedeutet. Im FE-Bericht Stickstoff 2013 wird daher auf S. 217 ein Abschneidekriterium von $0,3 \mathrm{~kg} \mathrm{~N} /\left(\mathrm{ha}^{\star} \mathrm{a}\right)$ und eine Bagatellschwelle von $3 \%$ des

69) Balla et al., Stickstoffeinträge in der FFH-VP 2014, siehe oben Fn. 25.

70) Wie bereits oben dargestellt: In „Balla et al., Stickstoffeinträge in der FFH-VP 2014" werden die Unsicherheiten von Modellen, nicht von Messungen für die Frage der statistischen Unterscheidbarkeit herangezogen.

71) Eine solche Kennzeichnung kann durch bereits enthaltene oder gesondert beigefügte Tracerstoffe erfolgen.

72) Grundlage dieses Teils ist die Arbeit „Untersuchung der Begründbarkeit von Abschneidekriterium und Bagatellschwelle des Forschungsberichts $2013 \mathrm{zu}$ straßenverkehrsbedingten Nährstoffeinträgen in empfindliche Biotope und des Stickstoffleitfadens Straße aus vegetationsökologischer Perspektive,“ Dipl.Biol. Friedrich Hacker, Mai 2021, die im gerichtlichen Verfahren BVerwG 9 A 1.21 vorgelegt wurde.

73) Bundesanstalt für Straßenwesen, Forschung Straßenbau und Straßenverkehrstechnik (BMVBS) 2013, Untersuchung und Bewertung von straßenverkehrsbedingten Nährstoffeinträgen in empfindliche Biotope, Bericht zum FE-Vorhaben 84.0102/2009 der Bundesanstalt für Straßenwesen, Bd. 1099 der Reihe „Forschung Straßenbau und Straßenverkehrstechnik, S. 218. 
Critical Loads empfohlen und in den Stickstoffleitfaden Straße 2019 auf S. 15 übernommen. ${ }^{74}$

Im FE-Bericht Stickstoff 2013, im Stickstoffleitfaden Straße 2019 und in den dort zitierten Quellen wird jedoch keine einzige wissenschaftliche Studie aufgeführt, die eine Wirkungsschwelle nachweist (6.2.1). Auch das vermeintliche Argument der Größe von Stickstoffgaben in wissenschaftlichen Studien trägt nicht (6.2.2). Festzustellen ist auBerdem, dass zumindest bei bestimmten Konstellationen auch Stickstoffeinträge $<0,3 \mathrm{~kg} \mathrm{~N} /\left(\mathrm{ha}^{\star} \mathrm{a}\right) \mathrm{zu}$ erheblichen negativen Effekten in gesetzlich geschützten Lebensräumen führen. In diesen Fällen ist die Untersuchung geeigneter Parameter erforderlich, um eine Beeinträchtigung sicher auszuschließen (6.2.3).

\subsubsection{Fehlender Nachweis einer Wirkungsschwelle}

In der Rechtsprechung ${ }^{75}$ wird argumentiert, dass empirische Untersuchungen an Straßen jenseits einer anhand des 3\%-Kriteriums ermittelten Grenze keine signifikanten schädlichen Effekte von Stickstoffeinträgen auf die Vegetation ergeben hätten.

Ein wissenschaftlicher Nachweis für eine empirische Wirkungsschwelle ist weder im FE-Bericht Stickstoff 2013 noch im Stickstoffleitfaden Straße 2019 enthalten. Es finden sich im FE-Bericht Stickstoff 2013 lediglich Verweise auf weitere Publikationen aus dessen Autorenkreis, die sich eingehender mit Abschneidekriterium und Bagatellschwelle befassen. ${ }^{76}$ Die früheste zitierte Publikation mit dem Vorschlag einer Bagatellschwelle von 3\% des CL ist ein Arbeitspapier der DEGES (Deutsche Einheit Fernstraßenplanungs- und -bau $\mathrm{GmbH}$ ) von $2007 .{ }^{77}$ Im Stickstoffleitfaden Straße 2019 wird auf S. 35 auf die Publikation ,Balla et al., Stickstoffeinträge in der FFH-VP 2014“78 verwiesen, in der originäre wissenschaftliche Studien ${ }^{79}$ etwas ausführlicher zitiert werden, aus denen das Abschneidekriterium von $0,3 \mathrm{~kg} \mathrm{~N} /\left(\mathrm{ha}^{\star} \mathrm{a}\right)$ und die Bagatellschwelle von $3 \%$ des CL angeblich ableitbar seien. Die zitierten Studien beziehen sich aber nur auf die Reichweite von Vegetationsveränderungen in Folge verkehrsbedingter Stickstoffdepositionen in natürlichen Lebensräumen. Es seien laut „Balla et al., Stickstoffeinträge in der FFH-VP 2014“, S. 48, in der Studie von Kirchner et al. 2007 nur bis zu einer maximalen Entfernung von $230 \mathrm{~m}$ zur Autobahn als Beeinträchtigung bewertbare Vegetationsveränderungen nachweisbar, und die zusätzliche Stickstoffdepositionsmenge betrug dort über $1 \mathrm{~kg} \mathrm{~N} /\left(\mathrm{ha} \mathrm{a}_{\mathrm{a}}\right)$. Tatsächlich wird in Kirchner et al. 2007 S. 106 dargelegt, dass der Einfluss der Autobahn auf die Bodenvegetation in den untersuchten Waldgebieten Forstenrieder Park und Otterfing zwischen dem 3. und 4. Transektpunkt in einer Entfernung von $230 \mathrm{~m}$ bis $520 \mathrm{~m}$ endet, also nicht bei den behaupteten $230 \mathrm{~m}$. Auf S. 55 wird in Kirchner et al. 2007 ausgeführt, dass die im Jahr 2004 entlang des Transekts Echinger Lohe durchgeführten Immissionsmessungen trotz des beträchtlichen Abstandes von $450 \mathrm{~m}$ zur Autobahn noch erhebliche, überwiegend verkehrsbedingte $\mathrm{NO}_{2}$-Konzentrationen ergaben. Da die Stickstoffdepositionsmenge im Quadrat mit der Entfernung zur Autobahn abnimmt, ist die tatsächliche Stickstoffdepositionsmenge am - hinsichtlich Entfernung und Stickstoffdeposition nicht näher bestimmten - Endpunkt der nachgewiesenen Vegetationsveränderungen zwischen $230 \mathrm{~m}$ bis $520 \mathrm{~m}$ Entfernung mit Sicherheit erheblich geringer als der Wert von $>1 \mathrm{~kg} \mathrm{~N} /$ (ha ${ }^{\star}$ a) an dem in ,Balla et al., Stickstoffeinträge in der FFH-VP 2014“ zitierten 3. Transektpunkt in $230 \mathrm{~m}$ Entfernung.

Anhand der irreführend zitierten Reichweite der Vegetationsveränderungen aus der Studie von Kirchner et al. 2007 wird im FE-Bericht Stickstoff 2013 auf S. 68 geschlussfolgert, dass geringere Stickstoffmengen als in $230 \mathrm{~m}$ Entfernung gemessen keine relevante Wirkung auf die Vegetation haben können. In Wirklichkeit wurde in Kirchner et al. 2007 an dem Punkt, bis zu dem die Vegetationsveränderungen reichten, die genaue Depositionsmenge gar nicht gemessen. In der Argumentation wird zusätzlich außer Acht gelassen, dass auch nicht untersucht wurde, ob in Summe aus verkehrsbedingter Stickstoffdeposition und Vorbelastung eine kritische N-Depositionsmenge in den Lebensräumen erreicht oder überschritten wurde. Die 2012 und 2017 wiederholten Untersuchungen ${ }^{80}$ im Naturwaldreservat Echinger Lohe zeigten hingegen eine räumlich fortschreitende Vegetationsveränderung. Die 2004 in Kirchner et al. 2007 erfasste Reichweite der Stickstoffeinträge stellte also keineswegs einen stabilen Zustand dar, der alle Langzeitfolgen der Stickstoffeinträge auf die Vegetation abbildete.

In einer anderen als Beleg angeführten Studie ${ }^{81}$ heißt es explizit: „The impacts of total nitrogen deposition are outside the scope of this review". ${ }^{2}$ Auch die einzige zitierte deutsche Studie Kirchner et al. $2007^{83}$ enthält keine systematische Erfassung der Vorbelastung und ist daher für die Ableitung einer Wirkschwelle ungeeignet. Diese Einschätzung wurde von einem an der Studie beteiligten Autor bestätigt. ${ }^{84}$

In „Balla et al., Stickstoffeinträge in der FFH-VP 2014“ wird auf S. 48 angegeben, dass der Wert für die angenommene Wirkungsschwelle vor allem auf einer Befragung von Fachleuten basiert, die eine Zusatzbelastung unter $0,3 \mathrm{~kg} \mathrm{~N} /\left(\mathrm{ha}^{\star} \mathrm{a}\right)$ übereinstimmend als nicht signifikant verändernd eingeschätzt haben sollen. Inhalte der Befragung, Beteiligte und Antworten sind nicht dokumentiert. Des Weiteren wird auf gleicher Seite 48 auf eine ,erweiterte Auswertung der aktuellen Literatur" im FE-Bericht Stickstoff 2013 verwiesen. Auch diese ist nicht dokumentiert. Nicht

74) FGSV Forschungsgesellschaft für Straßen- und Verkehrswesen 2019, Hinweise zur Prüfung von Stickstoffeinträgen in der FFHVerträglichkeitsprüfung - Stickstoffleitfaden Straße, S. 15

75) BVerwG, Urt. v. 23.4.2014 - 9 A 25.12, NuR 2014, 706, Rdnr. 45, mit Verweis auf FE-Bericht Stickstoff 2013, S. 217, und auf Balla et al., Stickstoffeinträge in der FFH-VP 2014, S. 6.

76) Uhl et al., 2007, Ermittlung und Bewertung der verkehrsbürtigen $\mathrm{N}$-Deposition in FFH-Gebieten. - Methodenvorschlag vor dem Hintergrund des BVerwG-Urteils vom 17.1.2007 (Westumfahrung Halle). - Arbeitspapier im Auftrag der DEGES; Uhl et al (2009): Ermittlung und Bewertung von Wirkungen durch Stickstoffdeposition auf Natura-2000-Gebiete in Deutschland, abrufbar unter https://www.foea.de/images/downloads/Beurteilung_von_Stickstoffwirkungen-COST729-Uhl-et-al.pdf; Balla et al., NuR, 2010, H. 9120101616, 625; Balla et.al., NuL 2010, 12/20102367, 371 .

77) Uhl et al., 2007, Ermittlung und Bewertung der verkehrsbürtigen $\mathrm{N}$-Deposition in FFH-Gebieten. - Methodenvorschlag vor dem Hintergrund des BVerwG-Urteils vom 17.1.2007 (Westumfahrung Halle). - Arbeitspapier im Auftrag der DEGES, S. 20.

78) Siehe oben Fn. 25.

79) Kirchner et al., 2006, Untersuchungen des Zustandes von Ökosystemen im Alpenvorland entlang von Gradienten des Stickstoffeintrags. Schlussbericht. Forschungsprojekt im Auftrag des Bayerischen Staatsministeriums für Umwelt, Gesundheit und Verbraucherschutz, StMUGV-Förderkennzeichen: 76a-8731.2 1999/1 GSF-FE-Nr.: 76425; Bignal et al., 2004, The ecological effects of diffuse air pollution from road transport. Engl. Nat. Res. Rep. 580.

80) Kudernatsch et al., 2019, Walddynamik in der „Echinger Lohe“, LWF aktuell. 122, 28-32.

81) Bignal, et al., 2004, The ecological effects of diffuse air pollution from road transport. Engl. Nat. Res. Rep. 580, 27.

82) Übersetzung: „Die Folgen der Stickstoff-Gesamtdeposition waren außerhalb des Rahmens der Untersuchung“.

83) Kirchner et al. 2006, Untersuchungen des Zustandes von Ökosystemen im Alpenvorland entlang von Gradienten des Stickstoffeintrags. Schlussbericht. StMUGV-Förderkennzeichen: 76a8731.2 - 1999/1 GSF-FE-Nr.: 76425

84) Bernhardt-Römermann (Inst. f. Ökologie u. Evolution. Univ. Jena), 2021, Fernmündliche Auskunft vom 9.9.2021 zu Kirchner et al., 2006. 
wiedergeben wird in der Diskussion zur Bagatellschwelle, dass in einer der zitierten Studien ${ }^{85}$ von einer vegetationswirksamen Reichweite straßenverkehrsbedingter NOxEmissionen bis $500 \mathrm{~m}$ ausgegangen wird.

Bei Tierhaltungsanlagen wird überwiegend Ammoniak $\left(\mathrm{NH}_{3}\right)$ emittiert, bei Verbrennungsprozessen dominieren Stickoxide (NOx). Im Stickstoffleitfaden Straße 2019 wird auf S. 51 ausgeführt, dass die toxische Wirkung (Critical Levels) von Stickstoffverbindungen, die in erster Linie $\mathrm{NH}_{3}$ betrifft, nur für epiphytische Flechten und Moose relevant sei. Diese Darstellung trifft nicht zu, auch einige der die FFH-Lebensraumtypen prägenden charakteristischen Gefäßpflanzen-, Moos- und Flechtenarten sind empfindlich gegen die toxische Wirkung von $\mathrm{NH}_{3}{ }^{86}$ Das Konzept der Critical Levels (CLE) wurde bei Abschneidekriterium und Bagatellgrenze bisher nicht hinreichend einbezogen.

Die erste umfassende Übersicht zu Studien über die Auswirkungen von Stickstoffdepositionen war der Review Cunha et al. $2002^{87}$, u.a. von Bobbink verfasst, dem Leiter der führenden Arbeitsgruppe zu CL. Die Studie Cunha et al. 2002 wurde von den Autoren des Forschungsberichts 2013 und von „Balla et al., Stickstoffeinträge in der FFH-VP 2014“" weder aufgegriffen noch berücksichtigt. Der Hinweis auf die zentrale Stellung der Studie Cunha et al. 2002 findet sich aber in der in „Balla et al., Stickstoffeinträge in der FFH-VP 2014“ zitierten Publikation Bignal et al. 2004, 41. Es stellt sich somit die Frage nach der Qualität der im FE-Bericht Stickstoff 2013 durchgeführten Quellenrecherche.

\subsubsection{Stickstoffgaben in Forschungsprojekten}

In der Rechtsprechung ${ }^{88}$ wird argumentiert, dass der $\mathrm{Ab}$ schneidewert von $0,3 \mathrm{~kg} \mathrm{~N} /\left(\mathrm{ha} \mathrm{a}^{\star}\right.$ ) deutlich unterhalb einer sicheren Ursache-Wirkungs-Relation liege, weil in wissenschaftlichen Studien mit Stickstoffabgaben in Stufen von mindestens 5 bis $10 \mathrm{~kg} \mathrm{~N} /\left(\mathrm{ha}^{\star} \mathrm{a}\right)$ gearbeitet worden sei.

Die Schwelle, ab der Stickstoffeinträge zu messbaren Auswirkungen auf die Biodiversität führen, hängt von der Empfindlichkeit des Lebensraums, der Vorbelastung, der Eintragsrate (kg N/ha), sowie Eintrags- und Wirkzeit ( $\star_{\mathrm{a}}$ ) ab. Das Produkt aus Eintragsrate mal Zeit bestimmt die Eintragssumme. Eintragsrate und Zeit substituieren sich, aber keineswegs linear. Unter kurzfristigen Auswirkungen werden Zeiträume $<5$ Jahre verstanden. Langfristige Wirkungen beziehen sich auf Zeiträume $>5$ Jahre. ${ }^{89}$ Langfristige Auswirkungen werden im Regelfall nach 15-30 Jahren nachweisbar. Im Ausnahmefall können bis zu 100 Jahre vergehen. ${ }^{90}$

Wissenschaftliche Studien sind in aller Regel auf $<5$ Jahre begrenzt. Ein erheblicher Teil wird in zwei bis drei Jahren über Dissertationen ausgeführt. Um in einer eigentlich zu kurzen Zeit Ergebnisse zu erzielen, wird die fehlende Zeit durch hohe Eintragsraten ersetzt. ${ }^{91} \mathrm{Die} \mathrm{Ab}-$ leitung einer Wirkungsschwelle aus den in solchen Studien gewählten Stickstoffgaben ist abwegig.

Entscheidender als die Größe der N-Gabe ist, ob in Summe mit der Vorbelastung kritische Schwellenwerte überschritten werden. Es liegen Studien vor, in denen der CL in Folge der experimentellen Stickstoffgabe um nur $1-2 \mathrm{~kg} \mathrm{~N} /\left(\mathrm{ha}^{\star} \mathrm{a}\right)$ überschritten wurde und Effekte auf die Vegetation schon nach nur 1-4 Jahren messbar waren. ${ }^{92}$ Es gibt daher keinen Grund anzunehmen, dass bei einer zu berücksichtigenden Wirkdauer von 15-30 Jahren nicht auch viel geringere Eintragsraten negative Effekte auf die Schutzgüter bewirken können.

Für den Nachweis langfristiger Wirkungen geringer Stickstoffeinträge sind Gradientenstudien und die wiederholte Erfassung von Dauerbeobachtungsflächen mit unterschiedlich hoher Vorbelastung besser geeignet als Experimente mit ein- oder mehrmaliger unnatürlich hoher N-Gabe. Die aus Gradientenstudien und Wiederholungs- untersuchungen abgeleiteten Spannen der noch einen Effekt auslösenden Stickstoffmenge sind teils viel kleiner. In Bobbink und Hettelingh ist in einer Studie für Waldlebensräume auf S. 208 eine Spanne von $7-8,5 \mathrm{~kg} \mathrm{~N} /\left(\mathrm{ha}^{\star} \mathrm{a}\right)$ und auf S. 209 aus einer anderen Studie ein Wert von 3,1 kg N/ (ha*a) angegeben. ${ }^{93}$ Die den Effekt auslösende Stickstoffmenge wird in den zitierten Studien demnach bis zu einer Genauigkeit von $1,5 \mathrm{~kg} \mathrm{~N} /\left(\mathrm{ha}^{\star} \mathrm{a}\right)$ bis $0,1 \mathrm{~kg} \mathrm{~N} /\left(\mathrm{ha}{ }^{\star} \mathrm{a}\right)$ angegeben.

\subsubsection{Schädigungen beim Überschreiten} von Schwellenwerten

Die Überschreitung von Schwellenwerten führt zur Schädigung der Lebensgemeinschaften charakteristischer Arten geschützter Lebensräume. Auch eine Schädigung von Anhang II - Arten der FFH-Richtlinie ist möglich. Schwellenwerte können bei entsprechender Vorbelastung bereits durch niedrige Zusatzeinträge überschritten werden. Es gibt daher keine unkritische Größe für zusätzliche Stickstoffeinträge, unterhalb derer Schäden ausgeschlossen werden können.

Die hauptsächlichen Wirkungen von Stickstoff (N) auf die Biodiversität von Landökosystemen sind Eutrophierung, Versauerung, direkte Blattschäden und Verstärkung anderer Stressfaktoren. ${ }^{94} \mathrm{Zu}$ den Stressfaktoren zählt auch ein Uberschuss an $\mathrm{N}$ in der Nahrung von charakteristischen Tierarten geschützter Lebensräume, beispielsweise Insekten. ${ }^{95}$ Ein erhöhter N-Gehalt in Pflanzen verstärkt die Dürreempfindlichkeit und Anfälligkeit gegen pathogene Organismen. Die Massenvermehrung von Parasiten wird gefördert. ${ }^{96}$ Für viele Moor- und Waldlebensräume sind in-

85) Hicks/Ashmore, 2005, Local impacts of road Vehicles. Implication of local ecological impacts of road vehicles for the exceedance of NOx annual limit values and air quality objectives. DEFRA Contract SPU 24, 56.

86) Sutton/Reis/Baker, 2009, Atmospheric Ammonia. Detecting emmison changes and enviromental impacts, 447.

87) Cunha et al., 2002, Whole ecosystem nitrogen manipulation: an updated review. JNCC Report 331.

88) BVerwG, Urt. v. 15.5.2019 - 7 C 27/17, NuR 2019, 763 , Rdnr. 38

89) Bobbink et al., 2002, Empirical nitrogen critical loads for natural and semi-natural ecosystems: 2002 update, 5; UBA, 2019, Einfluss von atmosphärischen Stickstoffeinträgen auf die Biodiversität von Insekten in terrestrischen Ökosystemen. Eine Literaturrecherche. Umweltbundesamt, FG II 4.3, 18.

90) Ministerium für Umwelt, Klima und Energiewirtschaft Baden-Württemberg, 2019, Ermittlung der Critical Levels und Critical Loads für Stickstoff Methodik für die Neufassung der Belastungsgrenzen für in Deutschland vorkommende Vegetationseinheiten (CL Bericht 2019), AG2 Critical Loads BadenWürttemberg, 32 .

91) Nordin, 2007, Nitrogen critical loads for terrestrial ecosystems in low deposition areas. An expert workshop of the Convention on Long-range Transboundary Air Pollution (LRTAP). 2930 March 2007 in Stockholm, Sweden. The Background document, 2 .

92) Strengbom et al., 2002, Parasitic fungus mediates change in nitrogen-exposed boreal forest vegetation. - Journal of Ecology 90, 61, 67; Nordin et al., 1998, Effects of simulated $\mathrm{N}$ deposition on understorey vegetation of a boreal coniferous forest. Functional Ecology. 12, 691, 699.

93) Bobbink/Hettelingh, 2011, Review and revision of empirical Critical Loads and dose-response relationships; Proceedings of an expert workshop, Noordwijkerhout, 23-25 June 2010, $204 \mathrm{f}$.

94) van Dobben et al., 2015, in: de Vries, Hetteling, Posch, Critical Loads and Dynamic Risk Assessments Nitrogen, Acidity and Metals in Terrestrial and Aquatic Ecosystems, 59.

95) Vogels et al., 2020, Imbalanced by overabundance Effects of nitrogen deposition on nutritional quality of producers and its subsequent effects on consumers, VBNE, 12.

96) Flückiger \& Braun, 2004, Ergebnisse aus Dauerbeobachtungsflächen von 1984 bis 2004, Bericht 2, Institut für Angewandte Pflanzenbiologie, $31 \mathrm{f}$. 
terne Grenzwerte für das Kohlenstoff-/Stickstoffverhältnis (C:N-Verhältnis) bekannt, deren Überschreitung den Erhalt des Lebensraums ausschließt. ${ }^{97,98}$ Für Heiden ist belegt, dass es kritische $N-G e h a l t e ~\left(N_{t}\right)$ für Heidekraut und einige Flechtenarten gibt, deren Überschreitung zum Zusammenbruch der Bestände dieser den Lebensraum prägenden Arten führt. ${ }^{99}$ Es existieren in der Regel für ein Schutzgut mehrere Schadschwellen auf unterschiedlichem Niveau. Jeder Schwellenwert ist als eigenständiger Tipping-Point zu verstehen, dessen Überschreitung zu einer (weiteren) Verschlechterung des Schutzguts führt.

Das Vorkommen charakteristischer Arten, die $\mathrm{Zu}-$ nahme von Stickstoffzeigerarten und die Abnahme der Vitalität von charakteristischen Baumarten, die eine Schlüsselfunktion für die Existenz des Lebensraum haben, sind teils linear abhängig vom N-Eintrag. ${ }^{100,101,102} \mathrm{Ob}$ Schwellenwerte durch zusätzliche N-Einträge überschritten und Beeinträchtigungen ausgelöst werden, hängt allein vom Abstand des Istwerts zum Schwellenwert, von der Größe der Zusatzbelastung und von deren Dauer ab. Sind für ein Schutzgut gesicherte Schwellenwerte belegt, muss durch geeignete Untersuchungen sichergestellt werden, dass ein Zusatzeintrag nicht zur Schwellenwertüberschreitung führt.

\section{Zusammenfassung}

Die Rechtsprechung zum Abschneidekriterium gründet weitgehend auf Annahmen aus dem technischen und dem vegetationsökologischen Bereich (Messuntergrenze, Zuordenbarkeit zu einer Quelle, Abgrenzbarkeit von der Hintergrundbelastung, fehlende schädliche Effekte, fehlende Ursache-Wirkungs-Relation). Die Rechtsprechung stützt sich hierfür insbesondere auf den FE-Bericht Stickstoff 2013 und den daraus entwickelten Stickstoffleitfaden Straße 2019 sowie auf die Veröffentlichung eines Autor^_ innenkollektivs aus dem Jahr 2014.

Es wurde gezeigt, dass keiner der Begründungsansätze aus den genannten Untersuchungen einer kritischen Überprüfung standhält. Die Argumentation in den Untersuchungen ist teilweise methodisch falsch (beispielsweise die Herleitung einer fehlenden statistischen Unterscheidbarkeit oder einer einheitlichen Messuntergrenze für verschiedene stickstoffhaltige Stoffverbindungen). Teilweise ist die Argumentation veraltet oder schlicht unvollständig (beispielsweise bzgl. der technischen Messuntergrenze). Teilweise werden Ergebnisse aus zitierten Arbeiten dargestellt, die diese Arbeiten überhaupt nicht enthalten (beispielsweise die Benennung von unteren Wirkschwellen für Stickstoffeinträge). Untersuchungen, die der Annahme des Abschneidekriteriums entgegenstehen, wurden dagegen nicht einbezogen.

Dies hat zur Konsequenz, dass das Abschneidekriterium von $0,3 \mathrm{~kg} \mathrm{~N} /\left(\mathrm{ha}^{\star} \mathrm{a}\right)$ in tatsächlicher Hinsicht falsch ist. Daraus folgt gleichzeitig, dass weder der FE-Bericht Stickstoff 2013 noch der Stickstoffleitfaden Straße 2019 (weiterhin) als Fachkonvention angesehen werden können.

Die fehlerhaften tatsächlichen Annahmen haben zunächst zur Konsequenz, dass einzelne rechtliche Fragen neu bewertet werden müssen. Das betrifft insbesondere die Vorgaben des EuGH, dass der sichere Nachweis einer Beeinträchtigung im Habitatschutz nicht erforderlich ist, eine Wahrscheinlichkeit reicht aus. ${ }^{103}$ Das BVerw ${ }^{104}$ entnimmt dem Abschneidekriterium, dass es die Grenze dieser Wahrscheinlichkeit markiere. Dies ist in tatsächlicher Hinsicht nicht der Fall, was die Anwendbarkeit der zitierten Judikatur des EuGH und der damit verbundenen Änderung der Nachweisführung nach sich zieht.

Die Fehler in den tatsächlichen Annahmen führen auBerdem dazu, dass das Abschneidekriterium von $0,3 \mathrm{~kg} \mathrm{~N} /$ $\left(\right.$ ha $\left.{ }^{\star} \mathrm{a}\right)$ nicht diejenige Grenze markiert, jenseits derer keine vernünftigen Zweifel am Ausbleiben von Schäden bestehen. ${ }^{105}$

Open Access. Dieser Artikel wird unter der Creative Commons Namensnennung 4.0 International Lizenz veröffentlicht, welche die Nutzung, Vervielfältigung, Bearbeitung, Verbreitung und Wiedergabe in jeglichem Medium und Format erlaubt, sofern Sie den/die ursprünglichen Autor(en) und die Quelle ordnungsgemäß nennen, einen Link zur Creative Commons Lizenz beifügen und angeben, ob Änderungen vorgenommen wurden.

Die in diesem Artikel enthaltenen Bilder und sonstiges Drittmaterial unterliegen ebenfalls der genannten Creative Commons Lizenz, sofern sich aus der Abbildungslegende nichts anderes ergibt. Sofern das betreffende Material nicht unter der genannten Creative Commons Lizenz steht und die betreffende Handlung nicht nach gesetzlichen Vorschriften erlaubt ist, ist für die oben aufgeführten Weiterverwendungen des Materials die Einwilligung des jeweiligen Rechteinhabers einzuholen.

Weitere Details zur Lizenz entnehmen Sie bitte der Lizenzinformation auf http://creativecommons.org/licenses/by/4.0/deed.de.

Open Access funding enabled and organized by Projekt DEAL.

97) Appl Veg Sci, 2017, 20, 115 f.; Fleck et al., Nitrogen Status and Dynamics in German Forest Soils, in: Wellbroke, Bolte, Status and Dynamics of Forests in Germany Results of the National Forest Monitoring, Ecological Studies 237, 2019, 130.

98) Succow \& Joosten, 2001, Landschaftsökologische Moorkunde, 78

99) Hicks et al., 2011, Nitrogen Deposition and Natura 2000: Science \& practice in determining environmental impacts. COST729, 107.

100) Bobbink/Hettelingh, 2011, Review and revision of empirical Critical Loads and dose-response relationships; Proceedings of an expert workshop, Noordwijkerhout, 23-25 June 2010, 177.

101) Remke, 2009, Impact of atmospheric nitrogen deposition on lichen-rich, coastal dune grasslands. PhD thesis, Radboud University Nijmegen, $41,43$.

102) Flückiger et al., 2011, Auswirkung erhöhter Stickstoffbelastung auf die Stabilität des Waldes. Synthesebericht. Institut für Angewandte Pflanzenbiologie, 35

103) EuGH, Urt. v. 14.1.2016 - C-399/14, NuR 2016, 119 Rdnr. 42; siehe oben 4.

104) BVerwG, Urt. v. 12.6.2019 - 9 A 2.18, juris Rdnr. 72.

105) Zu diesem Maßstab siehe EuGH, Urt. v. 7.9.2004 - C-127/02, Slg. 2004, I-7405, Rdnr. 59 und 61; BVerwG, Urt. v. 17.1.20079 A 20.05, BVerwGE 128, 1 Rdnr. 56; BVerwG, Urt. v. 11. 8.2016 - 7 A 1.15, BVerwGE 156, 20 Rdnr. 67). 